\title{
Combinatorics and topology of the Robinson tiling
}

\author{
Franz Gähler ${ }^{\mathrm{a}}$, Antoine Julien ${ }^{\mathrm{b}}$, Jean Savinien ${ }^{\mathrm{c}}$. \\ aniversity of Bielefeld \\ ${ }^{\mathrm{b}}$ University of Victoria-PIMS \\ ${ }^{\mathrm{c}}$ Université de Metz
}

\begin{abstract}
We study the space of all tilings which can be obtained using the Robinson tiles (this is a two-dimensional subshift of finite type). We prove that it has a unique minimal subshift, and describe it by means of a substitution. This description allows to compute its cohomology groups, and prove that it is a model set.

\section{Résumé}

Combinatoire et topologie des pavages de Robinson. Nous étudions l'espace de tous les pavages qui peuvent s'obtenir à partir des tuiles de Robinson (il s'agit d'un sous-décalage de type fini). Cet espace contient un unique sous-espace minimal, que nous décrivons par le biais d'une substitution. En conséquence, il est possible de calculer les groupes de cohomologie associés, et de montrer qu'il s'agit d'un pavage de coupe et projection.
\end{abstract}

\section{Version française abrégée}

C'est en 1971 que Robinson introduit l'ensemble de tuiles qui porte son nom. Un «pavage de Robinson» est un pavage que l'on peut obtenir à partir des tuiles de la figure 1 (ainsi que leurs images par rotation et reflexion). Les pavages de Robinson doivent en outre respecter les règles suivantes : les tuiles doivent se rencontrer face-à-face, et les flèches doivent rencontrer des lignes; par ailleurs, dans une colonne sur deux et une ligne sur deux, une tuile sur deux est de type (a) (voir fig. 1), sans restriction a priori sur son orientation. Les tuiles de type (a) sont appelées des « carrefours ».

Formellement, un pavage est une décoration de $\mathbf{Z}^{2}$ : à chaque élément du réseau correspond une tuile dans une orientation donnée. Ainsi, un pavage est un élément de $\mathcal{A}^{\mathbf{Z}^{2}}$, où $\mathcal{A}$ est l'ensemble des tuiles de Robinson. On note $\Xi$ l'ensemble des pavages de Robinson. C'est un sous-décalage de $\mathcal{A}^{\mathbf{Z}^{2}}$, c'est-à-dire un sous-ensemble fermé (donc compact), et invariant sous l'action de $\mathbf{Z}^{2}$ par décalage (translation). Un point

\footnotetext{
Email addresses: gaehler@math.uni-bielefeld.de (Franz Gähler), antoinej@uvic.ca (Antoine Julien), savinien@univ-metz.fr (Jean Savinien).
} 
important est que cet espace est non vide, et ne contient aucune période (voir figure 4 pour un amas de taille $7 \times 7$ ).

L'espace des pavages de Robinson n'est pas minimal, mais contient un unique sous-espace minimal, noté $\Xi_{\min }$. Le théorème principal de cet article s'énonce ainsi.

Théorème 1 Il existe une substitution (voir ci-dessous) $\tilde{\omega}$ telle que l'espace de pavages associé à la substitution $\Xi_{\tilde{\omega}}$ est topologiquement conjugué à $\Xi_{\min }$ : il existe un homéomorphisme entre ces deux espaces qui commute aux actions.

Dans cet article, une substitution $\omega$ est une application qui associe a chaque tuile un carré de $2 \times 2$ tuiles. L'itération d'une substitution produit une suite d'amas de taille croissante, et par un passage à la limite adéquat, produit un pavage $T$. On appelle $\Xi_{\omega}$ le plus petit sous-décalage de $\mathcal{B}^{\mathbf{Z}^{2}}$ qui contient $T$. Sous certaines conditions qui sont satisfaites ici, l'espace $\Xi_{\omega}$ ne dépend que de $\omega$, et pas de $T$. Il est de plus minimal et sans période.

La preuve du théorème ci-dessus se fait en deux parties. D'abord, on exhibe une substitution $\omega$, décrite en figure 3. Notons $\Theta$ l'application « oubli » $\mathcal{A} \rightarrow \mathcal{A} \cup\{$ blanche\}, qui est l'identité lorsque restreinte aux carrefours, et envoie toutes les autres tuiles sur la tuile blanche. Alors cette application s'étend aux pavages, et définit une application $\Xi_{\min } \rightarrow \Xi_{\Theta}$. Alors $\Xi_{\omega}$ et $\Xi_{\Theta}$ sont topologiquement conjugués. En d'autres termes, la substitution $\omega$ décrit entièrement l'emplacement des carrefours sur les pavages de Robinson. Cela dit, certains pavages de Robinson ne sont pas entièrement déterminés par la donnée de tous leurs carrefours. Ainsi, $\Xi_{\omega}$ n'est pas conjugué à $\Xi_{\min }$.

La seconde étape est donc de décorer la substitution $\omega$ en une substitution 《augmentée », notée $\tilde{\omega}$, de sorte que les pavages obtenus par la nouvelle substitution décrivent l'emplacement des carrefours, mais aussi des autres tuiles qui composent les pavages de Robinson (les carrefours sont reliés par des lignes simples ou doubles). Le processus de décoration est illustré en partie par la figure 5, qui décrit comment encoder la position des lignes simples (ce sont les lignes fléchées des tuiles (b) et (c), et les lignes non fléchées des tuiles (b) et (c), voir figure 1).

La conjugaison entre $\Xi_{\min }$ et $\Xi_{\tilde{\omega}}$ est construite explicitement par des dérivations locales. On donne une manière de re-coder un pavage de $\Xi_{\min }$ en terme des tuiles de $\tilde{\omega}$, et réciproquement.

Ce résultat a deux applications notables. Tout d'abord, une telle description permet de calculer des invariants topologiques pour l'espace $\Xi_{\min }$, en utilisant les méthodes d'Anderson et Putnam [1]. Si on note $\Omega$ la suspension de $\Xi_{\min }$, on a :

$$
\check{\mathrm{H}}^{2}(\Omega)=\mathbf{Z}[1 / 4] \oplus(\mathbf{Z}[1 / 2])^{10} \oplus \mathbf{Z}^{8} \oplus \mathbf{Z} / 4 \mathbf{Z} ; \quad \check{\mathrm{H}}^{1}(\Omega)=(\mathbf{Z}[1 / 2])^{2} \oplus \mathbf{Z} ; \quad \check{\mathrm{H}}^{0}(\Omega)=\mathbf{Z} .
$$

Par ailleurs, comme l'espace (minimal) des pavages de Robinson est substitutif, et chaque element contient un sous-ensemble périodique de carrefours, on peut appliquer un théorème de Lee et Moody [4, théorème 3], qui implique que $\Xi_{\min }$ peut être décrit par la méthode de coupe et projection. En particulier, le spectre de diffraction est purement ponctuel.

Avant de conclure, notons qu'une substitution plus simple, mais avec recouvrement de tuiles, et décrivant les mêmes pavages de Robinson, a été découverte indépendemment par Joan Taylor (communication privée). Cette substitution permet aussi de calculer la cohomologie, et donne les mêmes résultats. 


\section{Introduction}

In 1971, Robinson [5] introduced his aperiodic set of tiles, in order to build a two-dimensional subshift of finite type with no periodic orbit. We also refer to the very comprehensive paper by Johnson and Madden [3]. The Robinson tiling space is the set of all tilings which can be built from the Robinson tiles as follows. Consider a set $\mathcal{A}$ of 28 symbols. These symbols are the tiles of Figure 1 , as well as their images under rotations and reflections. Then, consider the subshift of finite type $\Xi \subset \mathcal{A}^{\mathbf{Z}^{2}}$ defined by:

(i) adjacency relations: two neighbouring tiles should meet in such a way that arrowheads of a tile meet arrowtails of its neighbour;

(ii) alternating cross rule: for all $x \in \Xi$, there is an element $n \in \mathbf{Z}^{2}$, such that for all $i \in(2 \mathbf{Z}) \oplus(2 \mathbf{Z})$, $x_{n+i}$ is a cross (see Figure 1). There may also be crosses at other positions.

a

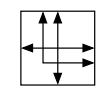

$\mathrm{b}$

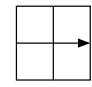

$\mathrm{c}$

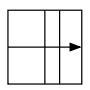

d

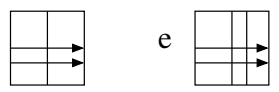

Figure 1. The Robinson tiles. The tiles of the first type (a) are called crosses.

Then, it is possible to show that $\Xi$ is not empty, and none of its elements has periods under the action of $\mathbf{Z}^{2}$ by translation. The key to this result is that any $x \in \Xi$ has a hierarchical structure: for all $n \in \mathbf{N}$, define a $n$-supercross as shown in Figure 4 (a 2-supercross is given as an example, on the left). Supercrosses are admissible under the adjacency rules. Therefore, by taking an appropriate union, one can build an element of $\Xi$. Conversely, the matching rules force supercrosses to appear in any admissible tiling. This gives a hierarchical structure which allows to prove non-periodicity of any $x \in \Xi$.

An increasing union of $n$-supercrosses is called an infinite order supertile. Since there are several ways of including a $n$-supercross in a $(n+1)$-supercross, there exist many different infinite order supertiles. They need not cover the whole plane. Given $x \in \Xi$, we have the following alternative:

(i) either $x$ is made of only one infinite order supertile;

(ii) or $x$ contains several (actually 2 or 4 ) infinite order supertiles.

Proposition 1.1 The subshift $\Xi$ contains a unique, minimal subspace, called $\Xi_{\min }$. Any element of $\Xi$ which follows alternative (i) is in $\Xi_{\min }$

To prove it, remark that any element of the tiling space contains $n$-supercrosses for all $n$. Therefore, elements which follow alternative (i) are accumulation points of any orbit of $\Xi$.

\section{A substitution}

We want to define a substitution map (see for example [1]) which describes the Robinson minimal tiling space. In our context, a substitution is a set of tiles $\mathcal{B}$, and a map $\omega: \mathcal{B} \rightarrow \mathcal{B}^{\{0,1\} \times\{0,1\}}$ which associates to every tile a $2 \times 2$ patch of tiles. Then the substitution tiling space $\Xi_{\omega}$ is the set of all elements $x \in \mathcal{B}^{\mathbf{Z}^{2}}$ such that any patch $x_{[n, n+k) \times[m, m+l)}$ of any size $k \times l$ appears (up to translation) as a subpatch of $\omega^{N}(t)$ for some integer $N$ and some tile $t$. It is a closed, shift-invariant subset.

Consider the tiles given in Figure 2, and the associated substitution given by Figure 3. One can check

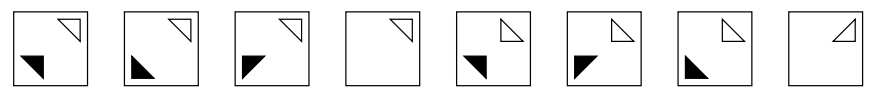

Figure 2. The tiles of the substitution. 

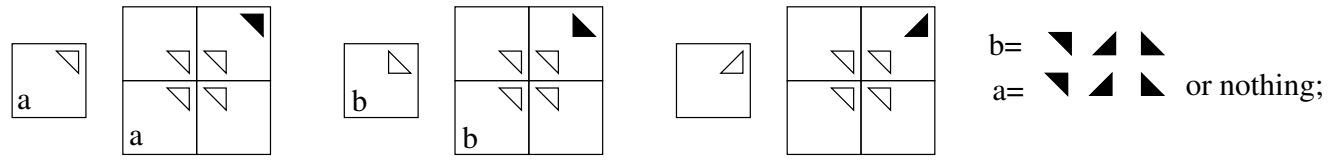

Figure 3. The substitution.

that this substitution is primitive: for any two tiles $a$ and $b$, the tile $b$ is contained in the $n$-th substitution of $a$, for $n$ big enough. It is known that tiling spaces associated with primitive substitutions are minimal. Theorem 2.1 The tiling space $\Xi_{\omega}$ associated with the substitution above is a factor of $\Xi_{\min }$, that is, there is an onto map $\phi: \Xi_{\min } \rightarrow \Xi_{\omega}$, which commutes with the shift.

We describe this factor more precisely in the next section. Then, we will prove the theorem. Let us mention without proof that this factor map is almost everywhere one-to-one. Moreover, we will explain below, by "decorating" the tiles and the substitution, how one can build a substitution $\tilde{\omega}$, whose tiling space is topologically conjugate to $\Xi_{\min }$.

\section{Local derivations}

A map $\phi: \mathcal{A}^{\mathbf{Z}^{2}} \rightarrow \mathcal{B}^{\mathbf{Z}^{2}}$ is called a local derivation (see [2]) if for any $x$, and any position $(i, j)$, the tile $\phi(x)_{i, j}$ only depends on the configuration of $x$ around $(i, j)$. That is, it only depends on the $x_{k, l}$, for $\|(k-i, l-j)\|<C$, where $C$ only depends on $\phi$. A local derivation is automatically continuous, and commutes with the shift.

Consider two minimal subshifts $\Xi_{\mathcal{A}} \subset \mathcal{A}^{\mathbf{Z}^{2}}$ and $\Xi_{\mathcal{B}} \subset \mathcal{B}^{\mathbf{Z}^{2}}$, and a local derivation $\phi$. If $x \in \Xi_{\mathcal{A}}$ and $\phi(x) \in \Xi_{\mathcal{B}}$, then by minimality, $\phi$ maps $\Xi_{\mathcal{A}}$ onto $\Xi_{\mathcal{B}}$, and therefore, it is a factor map. If there are two local derivations $\Xi_{\mathcal{A}} \rightarrow \Xi_{\mathcal{B}}$ and $\Xi_{\mathcal{B}} \rightarrow \Xi_{\mathcal{A}}$ which are inverse of each other, then the two subshifts are topologically conjugate. In order to prove Theorem 2.1 , we need to define a local derivation $\Xi_{\text {min }} \rightarrow \mathcal{B}^{\mathbf{Z}^{2}}$, and then prove that at least one point of $\Xi_{\min }$ is mapped to $\Xi_{\omega}$.
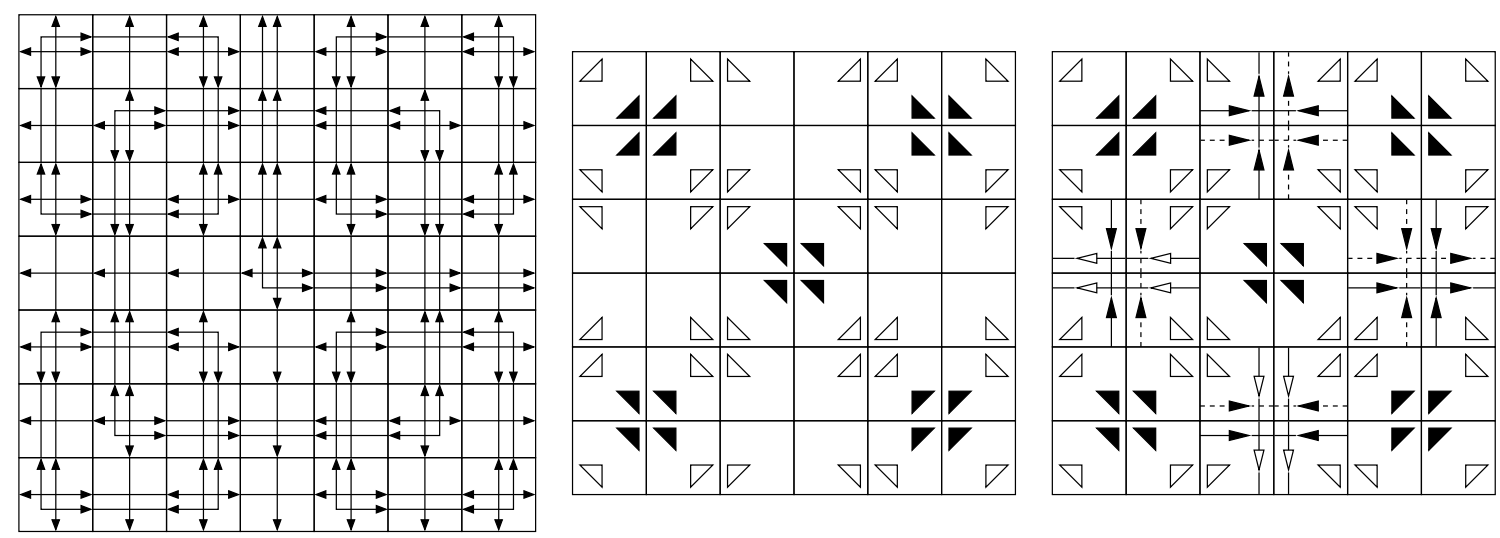

Figure 4. On the left, a 2-super-cross: it is made of four 1-super-crosses, and one cross in the middle. The middle cross is connected to lines which extend to the boundary of the super-cross: the supercross behaves like a cross of bigger size. In the middle, the image of this super-cross by the local derivation $\phi$ is shown. On the right, a fully decorated version of this super-cross (see Section 4) is given.

This local derivation is a composition of two maps $\phi=\phi_{2} \circ \phi_{1}$. The map $\phi_{1}$ is a "forgetful" map, which only remembers the position and orientation of the crosses. The map $\phi_{2}$ breaks the tiles and recomposes 
them. An example of a patch together with its image by $\phi$ is given by Figure 4 . More precisely, $\phi_{1}$ is defined as follows (note that whether a cross is subject or not to the alternating cross rule is a local information):

- The image of a cross which is subject to the alternating cross rule is a tile decorated with empty triangles which remember the orientation of the cross;

- The image of any other cross is a tile decorated with solid black triangles, which remember the orientation of the cross;

- The image of any other tile is a blank (empty) tile.

Remark that in a super-cross, every row and every column contains at least one cross. Therefore, all the tiles in a super-cross are entirely determined by the positions of the crosses. For this reason, the "forgetful" derivation described above is one-to-one when restricted to the set of Robinson tilings which follow alternative (i).

The map $\phi_{2}$ is defined by cutting all tiles obtained above in four, and recomposing them, so that the new tiles are now made of four pieces of four different previous tiles. The set of tiles which can be obtained is exactly $\mathcal{B}$, on which the substitution is defined. This derivation is of course invertible, by cutting tiles again and recomposing them (see Figure 4). We call $\phi$ the composition of $\phi_{1}$ and $\phi_{2}$.

In order to prove that $\phi$ maps $\Xi_{\min }$ to $\Xi_{\omega}$, we need to iterate the substitution $\omega$ on a tile. Remark that for any tile $t, \omega(t)$ contains the image of a cross under the local derivation. By iteration, one proves that $\omega^{n}(t)$ contains a $(n-1)$-super-cross (0-super-crosses being simply crosses). Therefore, the image under the local derivation of any Robinson tiling made of a single infinite order super-cross is contained in $\Xi_{\omega}$. We conclude, using minimality, that $\Xi_{\omega}$ is a factor of $\Xi_{\min }$.

\section{Decorating the substitution}

The idea is now to decorate tiles of $\mathcal{B}$ to get a set of tiles $\tilde{\mathcal{B}}$, and a substitution $\tilde{\omega}$ on it. If we call $\psi$ the map $\tilde{\mathcal{B}} \rightarrow \mathcal{B}$ which forgets the decorations, we require that $\psi \circ \tilde{\omega}=\omega \circ \psi$ (so that $\omega$ is induced by $\tilde{\omega}$ on undecorated tiles). Then, $\psi$ induces a local derivation $\Xi_{\tilde{\omega}} \rightarrow \Xi_{\omega}$. The augmented substitution $\tilde{\omega}$ should be defined in such a way that the two spaces $\Xi_{\min }$ and $\Xi_{\tilde{\omega}}$-of which $\Xi_{\omega}$ is a factor-are conjugate.

Note that $\Xi_{\omega}$ and $\Xi_{\min }$ cannot be conjugate to each other: tilings containing more than one infinite order supertile in $\Xi_{\text {min }}$ are mapped to tilings in $\Xi_{\omega}$ which may have several pre-images. The reason is that in such a tiling, there may be one row or one column in which there is no cross. The map $\phi$ forgets everything about such a line.

In order to get a space which is conjugate to $\Xi_{\min }$, we need to keep track in $\tilde{\omega}$ of the lines which connect crosses (simple lines, double lines, together with arrows orienting them).

Keeping track of simple lines. We describe how to produce decorations to keep track of simple lines (arrowed lines in tiles of type (b) and (c), and non-arrowed lines in tiles of type (b) and (d), see Fig. 1). Simple lines connect crosses which are "back-to-back". Therefore, we add decorations to the tiles in $\tilde{\mathcal{B}}$ in order to remember this fact (see Figure 5). Next, we decide how to substitute a decorated tile. This creates new decorated tiles which are not yet in $\tilde{\mathcal{B}}$. Then, we decide how these decorated tiles substitute. We iterate this process until no new tile is created. Finally, we discard any tile which doesn't appear any more in the eventual range of our decorated substitution.

In order to have a fully decorated substitution, we also need to keep track of double lines. See Figure 4 (patch on the right) for an example of a fully decorated patch. The decorated substitution $\tilde{\omega}$ requires 208 different tiles, up to translation. 

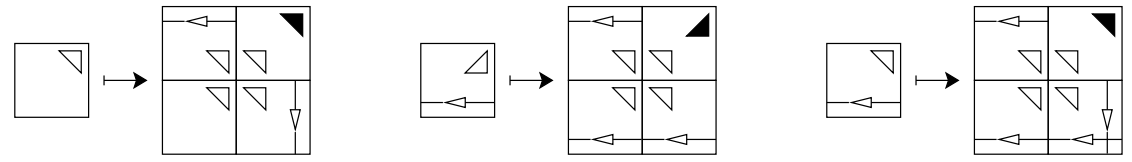

Figure 5. Single lines are coded by white-arrowed lines. In the first substitution, arrows were added near two edges. If such a patch occurs in a tiling $y \in \Xi_{\omega}$, then any $x \in \Xi_{\text {min }}$ which maps to $y$ will have a cross at the position of the black triangle. So, the white-arrowed lines in $y$ correspond to single lines in $x$. This creates new tiles. In the middle, the substitution of the lower-right tile of the first patch is shown, and on the right, the substitution of the lower-left tile of the second patch.

Theorem 4.1 Call $\tilde{\omega}$ the substitution defined on decorated tiles. Then the subshifts $\Xi_{\min }$ and $\Xi_{\tilde{\omega}}$ are topologically conjugate.

To prove this, we have to find rules to associate a tile of the Robinson tiling to any $2 \times 2$ patch of decorated tiles. This gives a local derivation which induces a factor map $\Xi_{\tilde{\omega}} \rightarrow \Xi_{\min }$. In the converse direction, we have to find a derivation from $2 \times 2$ Robinson patches to decorated tiles. The two derivations must be inverses of each other, in the sense that the image of any admissible $3 \times 3$ patch of a Robinson tiling under the composition of the two derivations is the central tile.

\section{Applications}

Since we now have a substitution $\tilde{\omega}$ on $\tilde{\mathcal{B}}$, it is possible to use the methods developed in [1] to compute the cohomology of the continuous hull of $\Xi_{\min }$. By continuous hull (or tiling space), we mean the suspension of the action of $\mathbf{Z}^{2}$ on $\Xi_{\min }$ : if $\sigma^{(n, m)}(x)$ is the image of $x$ by the shift, define:

$$
\Omega=\left(\Xi_{\min } \times \mathbf{R}^{2}\right) /\left\{\left(x,\left(t_{1}, t_{2}\right)\right) \sim\left(\sigma^{(n, m)}(x),\left(t_{1}-n, t_{2}-m\right)\right)\right\} .
$$

Given a substitution, one can associate a finite $\mathrm{CW}$-complex $\Gamma$, with 2-cells being (the interior of) the tiles. Then, the tiling space is homeomorphic to the inverse limit of $\Gamma$ under a map induced by the substitution. Using a computer program, it was possible to determine this complex, (adjacencies, etc.), compute its Cech cohomology, and the map induced by the substitution on cohomology.

Theorem 5.1 The cohomology groups of the Robinson minimal tiling space are:

$$
\mathrm{H}^{2}(\Omega)=\mathbf{Z}[1 / 4] \oplus(\mathbf{Z}[1 / 2])^{10} \oplus \mathbf{Z}^{8} \oplus \mathbf{Z} / 4 \mathbf{Z} ; \quad \mathrm{H}^{1}(\Omega)=(\mathbf{Z}[1 / 2])^{2} \oplus \mathbf{Z} ; \quad \mathrm{H}^{0}(\Omega)=\mathbf{Z} .
$$

Another consequence of the fact that the Robinson tiling can be described by a (lattice) substitution is that it is a model set. As every element of the hull contains a lattice periodic subset of crosses, this follows from Theorem 3 of [4]. As a further consequence, it also implies that every tiling in the hull has pure point diffraction spectrum.

Before concluding, let us mention that a somewhat simpler substitution, although with overlapping tiles, has been found independently by Joan Taylor (private communication). Also that substitution can be used to compute the cohomology, and gives the same results. 


\section{Appendix A. Complements to the article}

This appendix presents arguments (and pictures) for several results which were outlined in the article.

\section{A.1. Non-minimality of the Robinson space}

Non-minimality of the set of all admissible Robinson tilings is illustrated by Figure A.1. It shows a Robinson tiling made of two infinite order supertiles, separated by an infinitely long line of tiles. The two infinite order supertiles can be "sheared": if one translates the bottom infinite-order supertile to the right by any multiple of two, the result is still admissible (and satisfies the alternating cross rule). However, such a sheared tiling is not in $\Xi_{\text {min }}$ : the patch of Figure A.1 cannot appear in a super-cross.

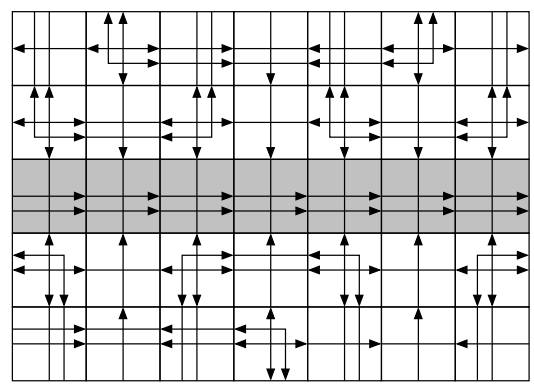

Figure A.1. The grey line extends to infinity on both sides. On top and bottom of it are two infinite order supertiles. One can shear the top and bottom infinite order supertile independently by any multiple of 2 and still get a Robinson tiling. However, such a tiling is not in $\Xi_{\min }$.

\section{A.2. The factor map $\Xi_{\min } \rightarrow \Xi_{\omega}$ is non-trivial}

We present several Robinson tilings which have the same image in $\Xi_{\omega}$. Such tilings are made of two infinite order supertiles, separated by an infinitely long line of tiles (of thickness 1 tile). This is described by Figure A.2.
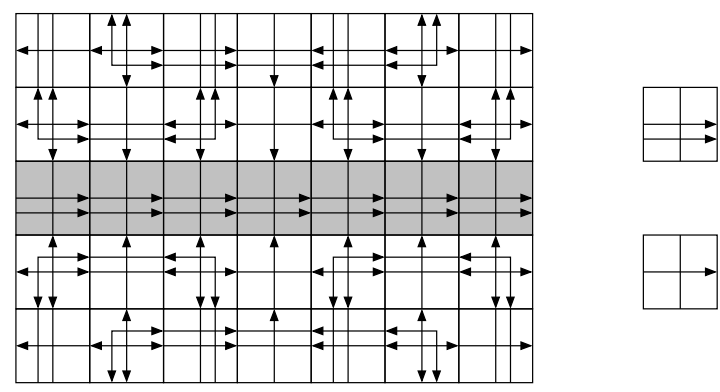

Figure A.2. The grey line extends to infinity on both sides. On top and bottom of it are two infinite order supertiles. If one changes the tiles on the grey line by any of the two tiles on the right (or their reflections), one gets different Robinson tilings, but their images in $\Xi_{\omega}$ are nevertheless the same.

In this picture, the grey row of tiles contains no cross. Therefore, its image in $\Xi_{\omega}$ is a row of blank tiles. If one changed the tiles which compose the grey row, the image would still be the same. Actually, one can check that there are six such Robinson tilings which have the same image in $\Xi_{\omega}$. 


\section{A.3. Decorated substitution}

We present here the decorated substitution. It is defined on the set of tiles given by Figure A.3 (as well as their images under rotation and reflection). The substitution itself is described by Figure A.4.

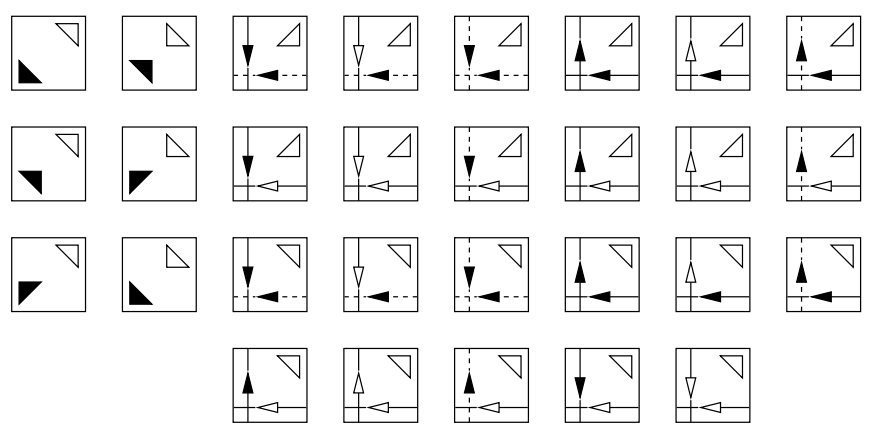

Figure A.3. Decorated tiles of $\tilde{\omega}$.
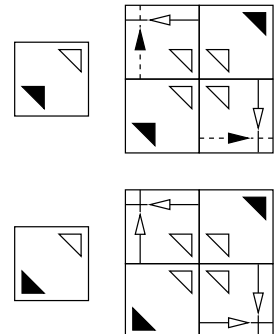
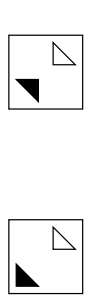
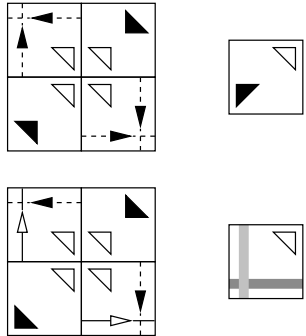
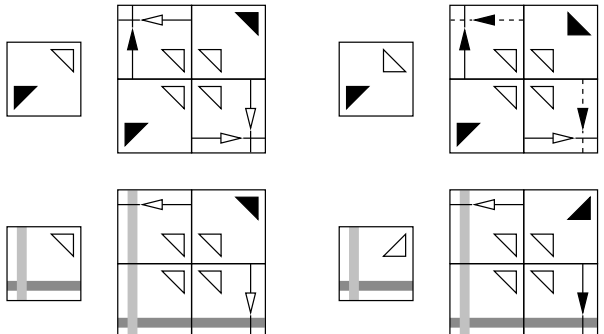
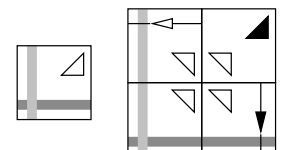

Figure A.4. The decorated substitution $\tilde{\omega}$. The two types of grey lines can be (possibly different) arrows of any type, a priori (black on plain line, black on dotted line or white, in any direction). The vertical (resp. horizontal) arrows in the substitution of a tile are of the same kind as the vertical (resp. horizontal) arrows in the tile. Note also that a posteriori, not all combinations of arrows are acceptable. See Figure A.3 for a list of all legal tiles.

A direct check shows that forgetting the decorations gives back the undecorated substitution $\omega$. It was checked, using a computer, that the substitution $\tilde{\omega}$ is primitive. As we already described, the augmented substitution appears to be a superposition of $\omega$, and of a one-dimensional substitution on arrows: the image of an arrow is two copies of itself (symbolically: $a \mapsto a a$ ).

\section{A.4. Local derivations}

The local derivations from $\Xi_{\min }$ to $\Xi_{\tilde{\omega}}$ and back can now easily be derived. Call $\mathcal{D}_{1}$ the local derivation $\Xi_{\min } \rightarrow \Xi_{\tilde{\omega}}$, and $\mathcal{D}_{2}$ the local derivation in the other direction. We describe these now. As a preliminary remark, we note that tiles of the Robinson tiling which are not crosses are defined by two features: vertical lines, and horizontal lines. Similarly, decorated tiles in $\tilde{\mathcal{B}}$ can be seen as the superposition of one, two or three features: a tile in $\mathcal{B}$, and possibly a vertical arrow, and possibly a horizontal arrow.

Let us first describe $\mathcal{D}_{1}$ : it is a map from $\mathcal{A}^{\{0,1\} \times\{0,1\}}$ to $\tilde{\mathcal{B}}$. We already described a map to $\mathcal{B}$, it is enough to describe how to add decorating features. This is done in Figure A.5.

The converse derivation $\mathcal{D}_{2}$ is defined similarly by a map from $\tilde{\mathcal{B}}^{\{0,1\} \times\{0,1\}}$ to $\mathcal{A}$. It is described in Figure A.6. 

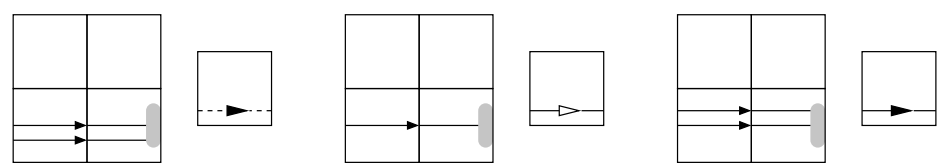

Figure A.5. The local derivation adding a decoration to the tiles of $\mathcal{B}$. If a $2 \times 2$ patch has an indicated feature (regardless of what the other tiles or vertical lines are), then the image tile has the indicated decoration. The grey ends of the lines can be arrowed, but don't need to: the image by the derivation is the same. Note that the rules for adding vertical decorations on the tiles can be deduced by rotating these pictures.
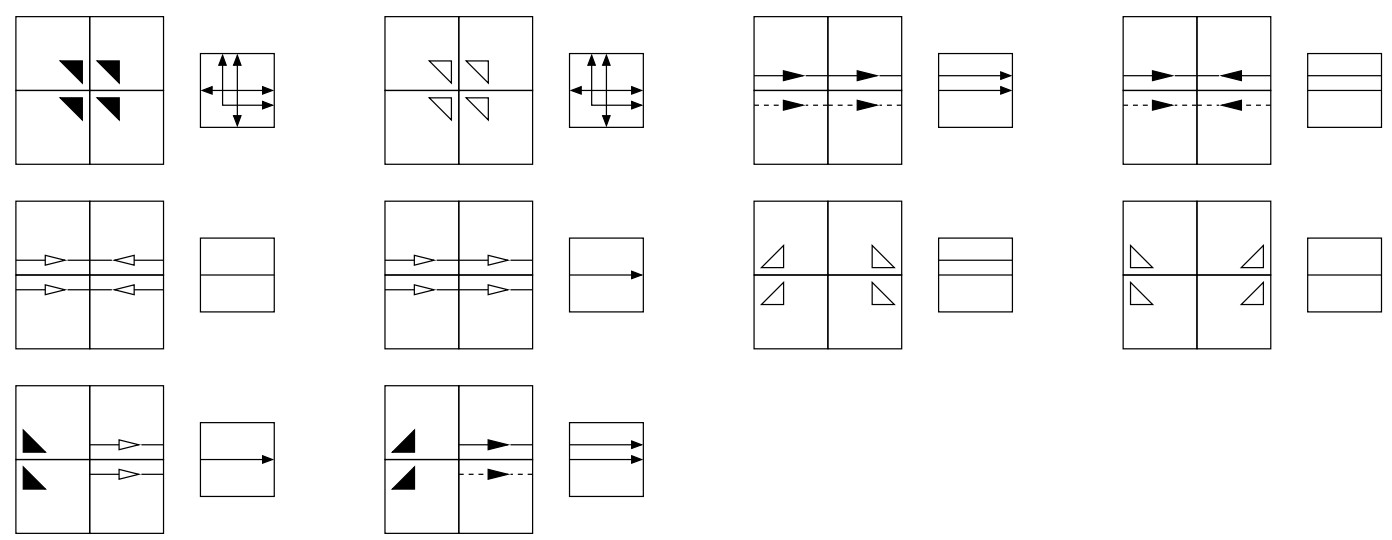

Figure A.6. The local derivation $\mathcal{D}_{2}$. If a $2 \times 2$ patch has one of the indicated features, then its image has the indicated horizontal lines. As in Figure A.5, the rules for the vertical lines can be obtained by rotating the pictures.

Figure A.7 shows three examples of local derivations using the rules above. Figure A.8 shows a local derivation on a $3 \times 3$ patch, composed with the local derivation backwards. The result is a single tile.
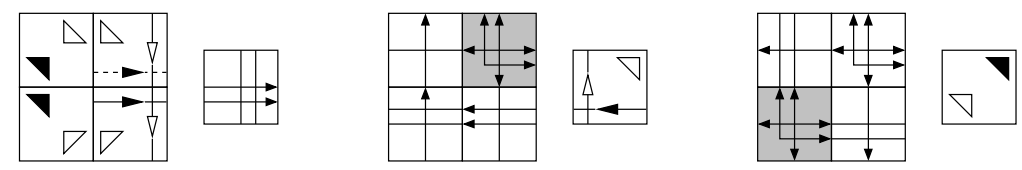

Figure A.7. Three examples of local derivations using the rules above. The tiles subject to the alternating cross rule were previously given a grey background (this change is a local derivation itself).
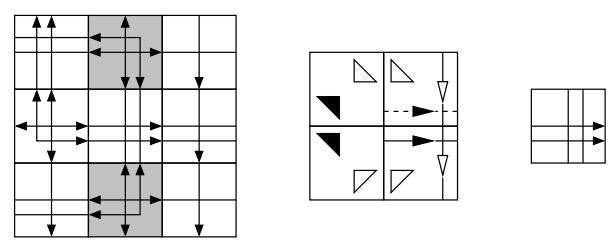

Figure A.8. The local derivations applied back and forth. Note that the final tile is the same as the central tile of the starting patch.

More generally, if the composition of these two derivations is applied on any $3 \times 3$ patch, the image is the centre tile of the original patch. This fact was checked on a computer. This proves that the factor maps induced by these derivations $\Xi_{\min } \rightarrow \Xi_{\tilde{\omega}}$ and $\Xi_{\tilde{\omega}} \rightarrow \Xi_{\min }$ are inverse of each other. Therefore, the spaces are topologically conjugate. 


\section{References}

[1] J. E. Anderson, I. F. Putnam, Topological invariants for substitution tilings and their associated $C^{*}$-algebras, Ergodic Theory Dynam. Systems 18 (1998) 509-537.

[2] M. Baake, M. Schlottmann, P. D. Jarvis, Quasiperiodic tilings with tenfold symmetry and equivalence with respect to local derivability, J. Phys. A: Math. Gen. 24 (1991) 4637-54.

[3] A. Johnson, K. Maddden, Putting the pieces together: understanding Robinson's nonperiodic tilings, College Math. J. 28 (1997) 172-181.

[4] J.-Y. Lee, R. V. Moody, Lattice substitution systems and model sets, Discrete Comput. Geom. 25 (2001), no. 2, $173-201$.

[5] R. Robinson, Undecidability and nonperiodicity for tilings of the plane, Invent. Math. 12 (1971) 177-209. 